\title{
Real and subjective lines and edges in the Bourdon illusion
}

\author{
JAMES T. WALKER and MATTHEW D. SHANK \\ University of Missouri-St. Louis, St. Louis, Missouri
}

\begin{abstract}
The Bourdon illusion consists of the apparent bending of a straight visual contour in the direction of a bent induction contour located nearby. The bent contour also appears partially straightened, or unbent, in the direction of the straight contour. These mutual attraction or assimilation effects are stronger between the edges of a solid figure than between the lines of an outline figure, whether the edges and lines consist of real or subjective contours. The present results are equally consistent with a cognitive model and a feature-detection model of these apparent displacement effects.
\end{abstract}

In the Bourdon illusion, two slender triangles are placed apex to apex with two of their long sides aligned. Figure 1 shows four examples of the Bourdon illusion. The illusion consists of the apparent inward bending of the collinear sides of the triangles.

Figures 1A and 1B show the usual forms of the Bourdon illusion (Bourdon, 1902; Jastrow, 1891; Rozvany \& Day, 1980; Wenderoth \& O'Connor, 1987a, 1987b; Wenderoth, O'Connor, \& Johnson, 1986a). In Figure 1A, where the triangles are composed of real lines, the collinear sides show little apparent bending. In Figure 1B, the real edges of the solid triangles produce a larger illusion. In Figures $1 \mathrm{C}$ and $1 \mathrm{D}$, where the triangles are composed of subjective lines and subjective edges, respectively, the illusion appears progressively greater. Indeed, we have found the Bourdon illusion to be substantially larger in subjective contours such as those in Figure 1D (Walker \& Shank, 1987).

Several other illusions have been demonstrated in subjective contours; these include the Ponzo (Farné, 1968), the Zöllner (Pastore, 1971), the Poggendorff (Goldstein \& Weintraub, 1972; Gregory, 1972; Meyer \& Garges, 1979), and perspective reversal in the Necker cube (Bradley \& Petry, 1977). For the most part, these illusions have been somewhat weaker in subjective contours.

Most illusions consist of a test portion that is distorted in some way by an induction portion. In most cases, induction contours repel test contours, as in the Zöllner illusion, for example. But in the Bourdon illusion, the collinear test contours, A and B in Figure 1 A, usually appear to be attracted by the objectively bent induction contours, C and D. However, repulsion effects sometimes occur in outline figures like Figure 1A, and in figures combining dashed and solid lines (e.g., Luckiesh, 1922/1965, p. 92; Rozvany \& Day, 1980; Wenderoth \& O'Connor, 1987b).

The authors' mailing address is Department of Psychology, University of Missouri-St. Louis, St. Louis, MO 63121.
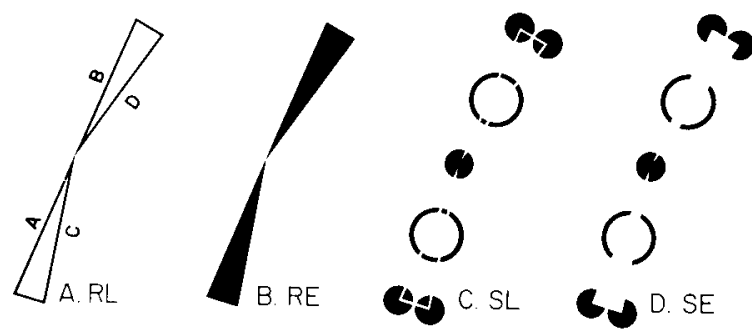

Figure 1. Illusions used in Experiment 1. (A) Real lines, RL; lines $A$ and $B$ are test contours, $C$ and $D$ induction contours. (B) Real edges, RE. (C) Subjective lines, SL. (D) Subjective edges, SE.

In most illusions, the effect-if any-of the test contours on the induction contours are ignored. In the Bourdon figures, the collinear test contours appear displaced toward the induction contours, and the induction contours appear displaced-partially unbent-in the direction of the test contours (Walker \& Shank, in press). Thus, there is mutual attraction or assimilation between the orientation of the test and induction contours in a Bourdon figure. We will refer to the apparent displacement of the collinear test contours as the Bourdon illusion, and to the apparent displacement of the bent induction contours as the unbending effect.

The Bourdon illusion readily lends itself to the study of interactions between real and subjective lines and edges because these kinds of visual contours can be combined in several ways in Bourdon figures. We have previously found the Bourdon illusion to be larger in figures in which the test and induction contours were both subjective than in figures in which both of these contours were real lines or real edges (Walker \& Shank, 1987). When real lines and subjective edges have been combined within figures, the subjective edges appeared strongly displaced but there was little or no apparent displacement of the real lines (Walker \& Shank, in press).

In the present study, we extend our earlier work on the Bourdon illusion and the unbending effect, using novel combinations of real and subjective lines and edges. The 
present experiments also provide further tests of a cognitive model and a feature-detection model of these contourdisplacement effects (Walker \& Shank, 1987).

The cognitive model of the Bourdon illusion and the unbending effect is based on the confusion of the orientation of the test and induction contours, whether these contours are real or subjective lines or edges. We first consider the nature of real contours. A real edge of a solid figure consists of a single luminance gradient, as in Figure 1B, whereas a real line consists of two luminance gradients, as in Figure 1A. Thus, lines contain more contour information than do edges. To the extent that two visual contours may be stronger or more compelling than a single contour, the orientation of lines should be more stable and less readily confused than the orientation of edges. Thus, the Bourdon illusion and the unbending effect should be larger in solid figures than in outlines.

Wenderoth and O'Connor (1987a, 1987b) proposed a similar explanation for the Bourdon illusion in solid figures. However, in outline figures, these workers found repulsion rather than attraction effects, and so did Wenderoth et al. (1986a). We will further consider the nature of these effects in outline figures in a later section.

The contours of a subjective figure can also consist of lines or edges. In Figure 1D, the solid subjective figure is bounded by subjective edges. This figure, like many similar examples, appears brighter than the background (Kanizsa, 1955, 1979). Thus, brightness contrast occurs across the edges of a Kanizsa-type subjective figure in physically homogeneous regions lacking any objective luminance gradients. There are, of course, physical $l u$ minance gradients within the sliced-pie inducing elements and at the line ends of the broken circles. But outside those immediate regions, the edges of the figure are associated with subjective brightness gradients.

The subjective edge in Figure 1D consists of a single brightness gradient, whereas a subjective line, as in Figure 1C, consists of two brightness gradients. Subjective lines therefore contain more contour information, and should be less readily confused than subjective edges. Thus, the Bourdon illusion and unbending effect should be greater in subjective edges than in subjective lines.

As we noted above, the Bourdon illusion and the unbending effect are greater in subjective contours than in real contours (Walker \& Shank, 1987, in press). Subjective contours appear weaker and less compelling than real contours (Halpern, Saltzman, Harrison, \& Widaman, 1983), and in addition, the just noticeable difference ( $j n d$ ) for the perceived orientation of subjective contours is greater than the jnd for the orientation of real contours (Vogels \& Orban, 1987). Thus, subjective contours should be more readily confused, and should produce greater apparent attraction or assimilation between the test and induction contours of a subjective Bourdon figure.

Before describing the feature-detection model of the Bourdon illusion and the unbending effect, we will further consider the nature of subjective contours. Several workers have proposed cognitive explanations of subjective contours (e.g., Gregory, 1972; Halpern \& Saltzman,
1983; Kanizsa, 1955, 1979; Rock \& Anson, 1979). In the cognitive view, subjective contours are the edges of subjective figures resulting from the apparent occlusion of parts of the inducing elements, as in Figure 1D.

Others have proposed brightness contrast as a possible explanation of subjective contours, but it is not entirely clear whether brightness contrast produces subjective contours or whether a subjective figure originating in some other way produces brightness contrast (e.g., Bradley \& Mates, 1985; Frisby \& Clatworthy, 1975; Ware, 1981). Nevertheless, brightness contrast is an empirical fact in the kinds of subjective figures used in the present study, whatever the origin of their subjective contours.

Feature detectors have been proposed as possible mechanisms that might account for subjective contours. For example, in Figures 1C and 1D, the corners of the subjective triangles are determined by objective luminance gradients where each apex partially occludes an inducing element. Thus, small portions of the lines and edges of these figures are real, and these real contours should stimulate local feature detectors such as the simple cells described by Hubel and Wiesel (1968). Smith and Over (1975) explained tilt aftereffects observed in subjective contours in terms of the adaptation of feature detectors. However, cognitive theorists have questioned the role of feature detectors, because subjective contours fail to occur in some cases in which feature detectors must surely be stimulated (e.g., Gregory, 1972; Halpern \& Saltzman, 1983).

The feature-detection model of the Bourdon illusion makes use of feature detectors encoding the orientation of lines and edges (e.g., Hubel \& Wiesel, 1968). Figure 2 illustrates the feature model we proposed earlier (Walker \& Shank, 1987). The horizontal axis in each panel represents a neural substrate that encodes spatial orientation,

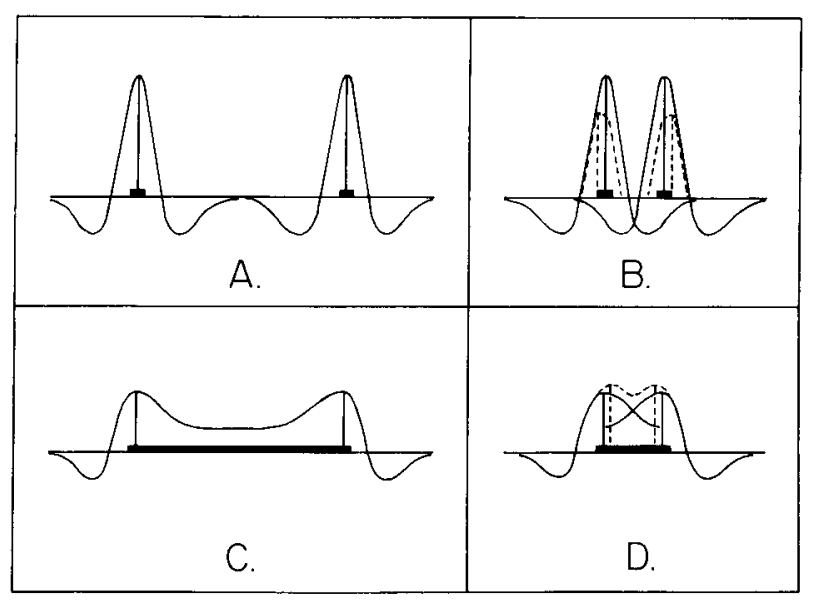

Figure 2. A model of interactions between lines and edges (from Walker \& Shank, 1987, p. 22). The horizontal axes represent spatial orientation and the vertical axes represent neural excitation and inhibition. (Top panels) Interactions between lines: (A) widely separated lines do not interact; (B) dotted lines show mutual repulsion of more closely spaced lines. (Bottom panels) Interactions between edges: (C) widely separated edges do not interact; (D) dotted lines show mutual attraction of more closely spaced edges. 
and the vertical axis represents neural excitation and inhibition.

Panel A in Figure 2 shows two real lines that are widely separated in orientation, and thus do not interact. In Panel B, the lines are closer in orientation, mutually inhibiting each other to some extent and resulting in mutual repulsion of their peaks of excitation, as the dotted lines show. Similar models of the apparent displacement of real contours in other illusions and figural aftereffects have been proposed (Coren \& Girgus, 1978, p. 90; Day, 1962; Ganz, 1966; Osgood \& Heyer, 1952; Smith \& Over, 1975; von Békésy, 1967; Wenderoth, O'Connor, \& Johnson, 1986b).

If the real lines in Panel B were closer together, with their excitatory peaks overlapping - a situation not illustrated here - the lines would display mutual attraction or assimilation rather than repulsion. As noted earlier, the Bourdon illusion is weaker in real outline figures, and indeed, repulsion effects are sometimes observed.

Panel C represents a solid figure with real edges. The peak of excitation at each edge represents a Mach band (Ratliff, 1965). Because of their wide separation, these excitatory peaks do not interact. In Panel D, the edges are closer together on the orientation dimension, and their excitatory peaks are mutually attracted, as the dotted lines show.

For real edges close enough to interact, the feature model predicts only attraction. Thus, the edges in Panel D are mutually attracted at the same separation at which the lines in Panel B repel each other. At some smaller separation, the lines would neither attract nor repel each other, but the edges would show mutual attraction. At still smaller separations, the lines would begin to show mutual attraction. Thus, real edges are expected to show mutual attraction if they are close enough to interact, whereas real lines are expected to show attraction at small separations and repulsion at larger separations.

The feature model can also explain interactions between subjective lines and edges if it is assumed that both subjective and real contours excite feature detectors. If the stimulation of feature detectors depends on the short objective luminance gradients associated with subjective contours, then these contours should stimulate feature detectors less strongly than real contours, or stimulate fewer feature detectors, or perhaps less sharply tuned feature detectors sensitive to a wider range of orientations. In all of these cases, the Bourdon illusion and the unbending effect should be larger in subjective contours. The model also predicts larger effects in subjective edges than in subjective lines, for the same reasons that larger effects are expected in real edges.

Three experiments were designed to test several predictions based on the above models. Experiment 1 compared the Bourdon illusion and the unbending effect in four illusion figures, each consisting of a single variety of contours, real lines or edges, or subjective lines or edges.
Experiment 2 measured these effects in figures by combining subjective lines and edges. Experiment 3 assessed the effects of line width in real outline figures.

\section{EXPERIMENT 1}

This experiment compared the Bourdon illusion and the unbending effect in the four illusions shown in Figure 1. Within each illusion, the test and induction contours both consisted of the same kind of contours: real lines (RL), real edges (RE), subjective lines (SL), and subjective edges (SE).

The cognitive model predicts the following order of the Bourdon illusion and the unbending effect across the set of illusion figures: $\mathrm{RL}<\mathrm{RE}<\mathrm{SL}<\mathrm{SE}$. Because lines-whether real or subjective-contain more contour information than edges, the cognitive model clearly predicts $\mathrm{RL}<\mathrm{RE}$ and $\mathrm{SL}<\mathrm{SE}$. If we assume that subjective contours, whether lines or edges, are less compelling and informative than real lines or edges, then the prediction of RE < SL follows, since subjective lines should be more readily confused than real edges. Indeed, Vogels and Orban (1987) found that the jnd for the orientation of a subjective edge was greater than that of a real line, lending credence to our present assumptions.

The feature model also predicts the same order of effects, and indeed, may predict this order somewhat more strongly. Real lines are more sharply defined than real edges, as shown in Figure 2. Analogously, we assume the same relationship between subjective lines and subjective edges. On these grounds, the feature model predicts $\mathrm{RL}<\mathrm{RE}$ and $\mathrm{SL}<\mathrm{SE}$.

Now consider the relationship between real edges and subjective lines. The real luminance gradients associated with subjective lines-that is, the objective luminance gradients of the sliced-pie inducing elements-are much shorter than the luminance gradients associated with real edges. It follows that real edges should be expected to stimulate feature detectors more strongly than subjective lines, and thus the feature model predicts RE $<$ SL perhaps more clearly than the cognitive model.

Both models predict the same order of effects across the set of illusion figures. Thus, neither model could be supported or disconfirmed independently of the other in the present experiment, although both models could be supported or both could be disconfirmed.

\footnotetext{
Method

Subjects. Eight men and 8 women, introductory psychology students at the University of Missouri-St. Louis, received extra course credit for their participation. The mean age was 18.44 years, and the range was from 18 to 22 .

Stimuli. Figure 1 shows the illusions used in the present experiment. Each illusion consisted of two isosceles triangles with longer sides of 5 in. $(12.7 \mathrm{~cm})$. The apex angles were $12.5^{\circ}$, and the figures were oriented as shown, with the collinear sides making an angle of $22.5^{\circ}$ with the vertical. This combination of apex angle and orien-
} 
tation produced the strongest Bourdon illusion in real contours (Rozvany \& Day, 1980), and was also used in our previous study of the illusion in subjective contours.

Figure 3 shows the comparison stimuli. The middle member of the comparison series was a straight line, $10 \mathrm{in} .(25.4 \mathrm{~cm})$ long and oriented $22.5^{\circ}$ from the vertical, like the collinear test contours of the illusions. The other comparison stimuli were bent $25^{\circ}$ and $50^{\circ}$. From left to right, the comparison stimuli were labeled $20 \mathrm{~L}, 10 \mathrm{~L}, 0,10 \mathrm{R}$, and $20 \mathrm{R}$.

The illusion figures were presented individually in a frontoparallel plane at a viewing distance of $2 \mathrm{~m}$. The illusions were presented approximately at eye level, and the comparison stimuli were presented directly below. Each illusion and comparison stimulus subtended a visual angle of about $7^{\circ}$ from end to end. Overhead fluorescent lights provided a luminance of $174 \mathrm{~cd} / \mathrm{m}^{2}$ on the white background of the illusions and $131 \mathrm{~cd} / \mathrm{m}^{2}$ on that of the comparison stimuli, as measured by a Macbeth illuminometer.

Procedure. The four illusions were presented to each subject in an individually randomized sequence. For each illusion, the order of test and induction judgments was also randomized individually.

In measuring the Bourdon illusion, the experimenter asked whether the collinear test contours appeared straight or bent. If the test contours appeared straight-that is, if there was no Bourdon illusion-then such a judgment was assigned a scale value of zero. If the test contours appeared bent, then the subject was instructed to choose the comparison stimulus that most closely matched the apparent bending of the test contours. This usually resulted in a choice of Comparison 0 . The subject then indicated whether the test contours appeared bent in the direction of $10 \mathrm{~L}$ or $10 \mathrm{R}$, and then chose a scale value between 0 and $10 \mathrm{~L}$ or between 0 and $10 \mathrm{R}$ to indicate the magnitude of apparent bending. When a larger amount of apparent bending resulted in a choice of Comparison 10R as the closest match, the subject indicated whether the test contours appeared bent in the direction of Comparison 0 or 20R, and then chose a scale value between $10 \mathrm{R}$ and 0 , or between $10 \mathrm{R}$ and $20 \mathrm{R}$ to indicate the perceived magnitude of bending. Apparent bending in the direction of the comparison lines on the right side of the display was taken as a positive Bourdon illusion.

The unbending effect was measured by similar procedures. If there was no apparent displacement of the bent induction contours, then the subject would match these contours to Comparison $10 R$. If the unbending effect occurred, then the subject would match the apparent bending of the induction contours to a scale value less than 10R. If the induction contours appeared displaced away from the test contours, then the subject would match the induction contours to a scale value greater than $10 \mathrm{R}$. Apparent displacement of the

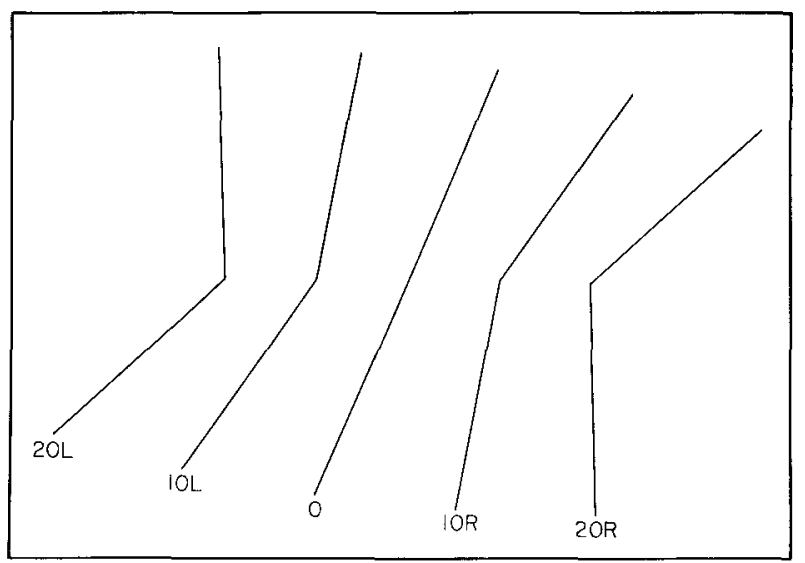

Figure 3. Comparison stimuli. Lines are progressively bent in $25^{\circ}$ increments.

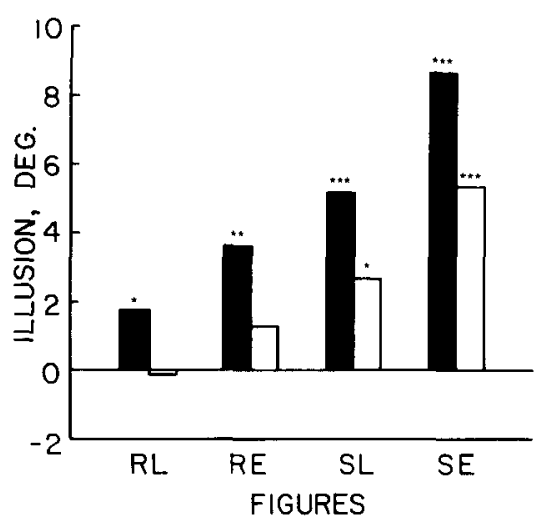

Figure 4. Results of Experiment 1. Mean Bourdon illusion (solid bars) and unbending effect (open bars) in degrees. Illusions are labeled as in Figure 1. Asterisks indicate values significantly different from zero: ${ }^{*} p<.05,{ }^{* *} p<.01,{ }^{* *} p<.001$.

induction contours toward the collinear test contours was taken as a positive unbending effect. These measurement procedures were used in our earlier studies of the Bourdon illusion and unbending effect (Walker \& Shank, 1987, in press).

We had previously observed an illusion of apparent displacement of the midpoints of the comparison stimuli. These midpoints were aligned horizontally, but they appeared higher on the right side of the comparison display. Therefore, after judging the Bourdon illusion and the unbending effect, each subject was asked whether the midpoints of the comparison lines appeared higher on the left or on the right side of the display.

\section{Results and Discussion}

Figure 4 shows the means of the Bourdon illusion and the unbending effect for each of the illusion figures. All of the figures produced Bourdon illusions that differed significantly from zero [values of $t(15)$ ranged from 2.91 , $p<.05$, to $9.73, p<.001]$. Only Figures SL and SE produced significant unbending effects [values of $t(15)$ were 2.40 and 7.06 , respectively, $p<.05$ and $p<$ $.001]$.

The results were subjected to a $2 \times 4$ repeated measures analysis of variance where the factors were kinds of contours (test and induction) and illusion figures (RL, RE, $\mathrm{SL}$, and SE). There were repeated measures on both factors. The Bourdon illusion was greater than the unbending effect, $4.77^{\circ}$ versus $2.27^{\circ}$, as shown by the significant effect of test versus induction contours $[F(1,15)=$ $14.55, p<.01]$. The main effect of the illusion figures was significant $[F(3,45)=23.04, p<.001]$, and there was no significant contour $\times$ figure interaction $(F<1)$.

The simple effects of the figures were significant for the Bourdon illusion and the unbending effect as well [both values of $F(3,88) \geq 10.16, p<.001]$. The error term used in testing these simple effects was a composite of the error terms for the main effects of figures and the contour $\times$ figure interaction, as recommended by Winer (1971).

Both models predict the same order of the Bourdon illusion and the unbending effect across the set of illusion 
figures, namely $\mathrm{RL}<\mathrm{RE}<\mathrm{SL}<\mathrm{SE}$, as in Figure 4. Under the assumptions of the models, we can scale the illusion figures in terms of the relative strength of the contour interactions underlying the apparent displacements, thus yielding ordinal measurements. However, neither model is sufficiently precise to quantify these contour processes on an interval or ratio scale, and thus our measurements do not meet the strict requirements of a trend analysis. Nevertheless, a failure to find a linear component in the simple effects of figures would call the models into question. As expected, the linear components of the simple effects of figures were significant for the Bourdon illusion and also for the unbending effect $[F(1,88)$ $=45.92$ and 29.60 , respectively, $p \mathrm{~s}<.001]$.

Neither the cognitive nor the feature model predicts any difference between the magnitude of the Bourdon illusion and the unbending effect, but in fact, the Bourdon illusion was significantly greater. This finding may relate to the difference between the orientations of the test and induction contours with respect to the vertical.

Rozvany and Day (1980) found the largest Bourdon illusion when their collinear test contours were oriented $22.5^{\circ}$ from the vertical. In an independent experiment, these workers measured the accuracy with which a single comparison line could be set to appear collinear with a test line presented at several orientations. This task was performed most accurately when the test line was vertical or horizontal, and least accurately when the test was oriented $22.5^{\circ}$ from the vertical, a finding related to the oblique effect observed in several other contexts (Appelle, 1972). Thus, acuity for orientation was poorest and the Bourdon illusion was greatest at $22.5^{\circ}$ from the vertical. Rozvany and Day attributed the greater Bourdon illusion at that orientation to the lower acuity for orientation.

In our present experiment, the test contours were oriented $22.5^{\circ}$ from the vertical. The lower and upper induction contours were, respectively, $10^{\circ}$ and $35^{\circ}$ from the vertical. Applying Rozvany and Day's (1980) findings, orientation acuity should have been poorer for the collinear test contours than for the bent induction contours. Thus, the Bourdon illusion should have been greater than the unbending effect, as was in fact the case.

Within the comparison stimuli, there was an illusion of vertical displacement of the midpoints of the bent com- parison lines. For 14 of the 16 subjects, the midpoints of these lines appeared higher on the right side of the display than on the left $(Z=2.75, p<.01)$. A similar illusion consistently occurred in similar comparison stimuli in our earlier studies of the Bourdon illusion (Walker \& Shank, 1987, in press).

The axes of all the comparison stimuli were tilted clockwise $22.5^{\circ}$ from the vertical, so it may be that the subjective vertical was also displaced in that direction. If the subjective horizontal were also rotated clockwise, then the horizontally aligned midpoints of the bent comparison lines would appear higher on the right side of the display, perhaps due to a frame-of-reference effect (see, e.g., Gibson \& Radner, 1937). It is also possible that some features of the Poggendorff illusion may apply here (see Coren $\&$ Girgus, 1978 , p. 60 , and Pressey \& den Heyer, 1968, for a Poggendorff variant that may apply).

Since the illusion in the comparison stimuli involves an apparent vertical displacement, as opposed to a displacement of orientation, there is no indication that such an effect interferes with measuring the Bourdon illusion or the unbending effect. Indeed, our present measurement of the Bourdon illusion in figure RE, $4.07^{\circ}$, compares closely with earlier measurements using adjustment procedures and comparable figures (e.g., $3.68^{\circ}$ in Rozvany \& Day, 1980 , and $3.62^{\circ}$ in Wenderoth et al., 1986a, Experiment 2 , chevron measurements, $10^{\circ}$ apex angle).

\section{EXPERIMENT 2}

In Experiment 1, the test and induction contours within each illusion were of the same kind, real or subjective lines or edges. Experiment 2 utilized illusions consisting of combinations of the subjective lines and edges shown in Figure 5.

In Figure 5A, the control figure, an imaginary line connecting the centers of the five collinear disks was not expected to show any apparent displacement, nor was the imaginary bent line connecting the other disks in this figure. All of the illusions in this experiment consisted of subjective lines and subjective edges in various combinations. Figure 5B, where the test and induction contours are subjective lines, is designated figure LL. In Figure 5C, designated figure LE, the test contours are

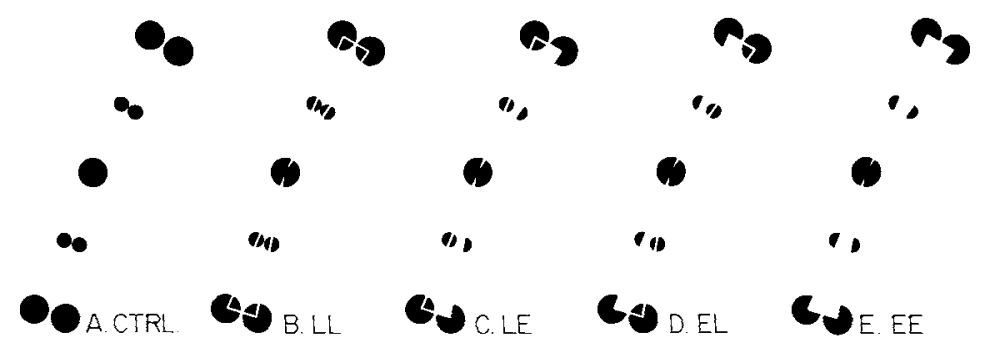

Figure 5. Subjective-contour illusions used in Experiment 2. (A) control figure; (B) test lines, induction lines, LL; (C) test lines, induction edges, LE; (D) test edges, induction lines, EL; (E) test edges, induction edges, EE. 
lines and the induction contours are edges. In Figure 5D, figure EL has test edges and induction lines. Finally in Figure 5E, figure EE has test edges and induction edges. In describing each of these figures, the first letter refers to the test contours and the second letter refers to the induction contours.

We now apply the cognitive model in predicting the magnitude of the Bourdon illusion in the present stimuli. A subjective line consists of two brightness gradients, and therefore contains more contour information than a subjective edge consisting of a single brightness gradient. In figure LL, there is a total of four brightness gradientstwo in the collinear test lines and two in the bent induction lines. There should be little confusion between the orientation of the contours in this figure, and little Bourdon illusion.

In figure LE, there are three brightness gradients, two in the test lines and one in the induction lines. This figure contains less contour information, and should produce greater confusion and a greater Bourdon illusion than figure LL. Figure EL also contains three brightness gradients, one in the test edge and two in the induction lines. Here, the test contours should be confused more readily with the induction contours, and thus figure EL should produce a greater Bourdon illusion than figure LE. Finally, figure EE should produce the greatest Bourdon illusion because there are only two brightness gradients here, one in the test edges and the other in the induction edges. Thus, the cognitive model predicts the following order of the Bourdon illusion across the present set of figures: $\mathrm{C}<\mathrm{LL}<\mathrm{LE}<\mathrm{EL}<\mathrm{EE}$.

For the unbending effect, the cognitive model predicts a somewhat different order. Figure LL should produce the smallest amount of unbending, and figure EE, the largest. But the subjective lines in the induction contours of figure EL should produce less unbending than the subjective edges in figure LE. Thus, the cognitive model predicts the following order of the unbending effects: $\mathrm{C}$ $<\mathrm{LL}<\mathrm{EL}<\mathrm{LE}<\mathrm{EE}$.

Considering the predictions of the feature model, if there is greater attraction between subjective edges than between subjective lines, then the smallest Bourdon illusion should occur in figure LL and the largest in figure EE. The subjective test lines in figure LE should show a smaller Bourdon illusion than the test edges in figure EL. Thus, the feature model predicts the same order of the Bourdon illusion as does the cognitive model, and the two models also predict the same order of the unbending effects.

However, we might argue that subjective lines excite feature detectors more strongly than subjective edges. In that case, the induction lines in figure EL might produce a greater Bourdon illusion in the test edges and the induction edges in figure $\mathrm{EE}$ might produce a smaller Bourdon illusion, contrary to the above predictions. Similarly, the subjective test lines in figure LE might produce a greater unbending effect in the induction edges, and the test edges in figure EE might produce a smaller unbend- ing effect, also contrary to the predictions above. Thus, we have less confidence in the predictions of the feature model in the present experiment, and more confidence in those of the cognitive model.

\section{Method}

Subjects. The subjects were 14 male and 4 female introductory psychology students. Their ages ranged from 18 to 22 years, with a mean of 19.28 .

Stimuli. The control figure and the illusions are described above. The comparison stimuli were the same as those used in Experiment 1 . The displays were the same size as in Experiment 1, and the viewing distance and levels of luminance were also the same.

Procedure. Except for the control judgments, the procedures were the same as those in the previous experiment. In judging the control figure, the subjects were instructed to draw an imaginary line connecting the centers of the five collinear disks (see Figure 5A), and to match the apparent bending of this imaginary line to a scale value on the comparison display. The subjects also judged the imaginary bent line connecting the other disks in the control figure. All of the figures, including the control figure, were presented in individually randomized sequences.

As in Experiment 1, after judging the Bourdon illusion and the unbending effect, each subject was asked whether the midpoints of the comparison lines appeared higher on the left or on the right side of the display.

\section{Results and Discussion}

Figure 6 shows the results. In the control figure, the means of the Bourdon illusion and the unbending effect were, respectively, $-0.42^{\circ}$ and $1.53^{\circ}$. Neither of these means differed significantly from zero (both values of $t<1$ ). Each of the illusion figures produced a significant Bourdon illusion [all values of $t(17) \geq 5.29$, $p s<.001$ ] , and a significant unbending effect [values of $t(17)$ ranged from $2.34(p<.05)$ to $5.24(p<.001)]$.

In the control figure, the size and placement of the black disks were the same as in the illusions, so the control figure differed from the illusions principally in terms of lacking subjective lines and edges. Another difference was that the control figure had complete disks whereas the illusion figures had sliced-pie disks. The absence of the Bourdon illusion and the unbending effect in the control

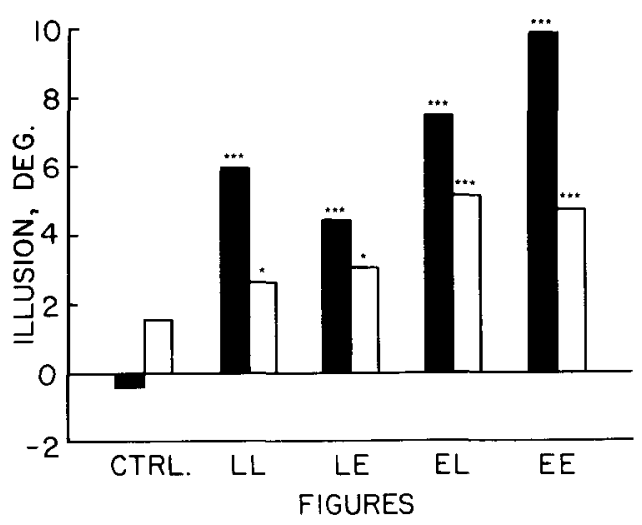

Figure 6. Results of Experiment 2. Bourdon illusion (solid bars) and unbending effect (open bars) in subjective-contour figures. Asterisks indicate significance levels: ${ }^{*} p<.05,{ }^{* *} p<.01,{ }^{* * *} p<.001$. 
figure suggests that these apparent displacements cannot be due merely to the global configuration of the black disks in the illusion figures, apart from their role in producing subjective lines and edges.

The results were subjected to a $2 \times 5$ repeated measures analysis of variance. The factors were kinds of contours (test and induction) and illusion figures (control, LL, LE, EL, and EE). There were repeated measures on both factors.

The effect of figures was significant $[F(4,68)=17.76$, $p<.001]$, the effect of test versus induction contours approached significance $[F(1,17)=3.12, p<.10]$, and there was a significant contour $\times$ figure interaction $[F(4,68)=4.09, p<.01]$. Thus, the simple effects of figures and contours are of interest.

In all four illusion figures, the Bourdon illusion was greater than the unbending effect. Pooling the simple effects of test versus induction contours for the illusion figures--excluding the control figure-the Bourdon illusion was greater than the unbending effect $[F(4,54)=$ $4.16, p<.01]$. The error term for this comparison was a composite of the error terms for testing the effects of contours and the contour $\times$ figure interaction in the analysis of variance (Winer, 1971).

Neither of our models predicts a difference between the Bourdon illusion and the unbending effect. As we suggested in Experiment 1, the orientations of the induction contours may have resulted in greater visual acuity for these contours, which may have led to a smaller unbending effect relative to the Bourdon illusion.

The cognitive model predicts the following order of the Bourdon illusion across the set of figures, namely $\mathrm{C}<$ $\mathrm{LL}<\mathrm{LE}<\mathrm{EL}<\mathrm{EE}$. (The feature model also predicts this order, though perhaps equivocally, as noted above.) Figure 6 lists the illusion figures in the predicted order of magnitude of the Bourdon illusion, and shows the observed results. The Bourdon illusion was greater in figure LL than in figure LE, representing a reversal of the predicted order.

For the unbending effect, the cognitive model predicts a different order across the set of figures, namely $\mathrm{C}<$ $\mathrm{LL}<\mathrm{EL}<\mathrm{LE}<\mathrm{EE}$. In fact, the unbending effect was greater in EL than in LE, and greater in EL than in $\mathrm{EE}$, which represents two reversals of the predicted order.

The models failed to predict precisely the observed orders of the Bourdon illusion and the unbending effect, since there was one reversal in one of these observed orders and two reversals in the other. However, the trend analyses below may provide more comprehensive tests of the observed orders. These analyses are subject to the same criticism as in Experiment 1. Since the models specify only the relative strength of the contour interactions in the illusion figures, the levels of the independent variable comprise only an ordinal scale.

The simple effects of figures were significant for the Bourdon illusion $[F(4,135)=18.38, p<.001]$ and also for the unbending effect $[F(4,135)=2.78, p<.01]$. As predicted, the linear component of the simple effect of figures was significant for the Bourdon illusion $[F(1,135)$ $=60.41, p<.001]$ and also for the unbending effect $[F(1,135)=5.74, p<.05]$.

In the comparison stimuli, the illusion was the same as in Experiment 1. The midpoints of the bent comparison lines appeared higher on the right side of the display to 14 of 17 subjects $(Z=2.43, p<.02)$. One subject's response to this illusion was not recorded because of experimenter error.

\section{EXPERIMENT 3}

This experiment was designed to assess the effects of line width on the Bourdon illusion and the unbending effect in figures with real test and induction contours. Figure 7 shows the illusions used in the present experiment, progressing from the narrow lines of Figure 7A, through the wider lines of Figures $7 \mathrm{~B}$ and $7 \mathrm{C}$, to the edges of the solid figure in Figure 7D.

The cognitive model predicts the same order of magnitude for the Bourdon illusion and the unbending effect, namely, $\mathrm{A}<\mathrm{B}<\mathrm{C}<\mathrm{D}$. Thus, both effects were expected to increase with increasing line width.

In figure $\mathrm{A}$, the cognitive model predicts little confusion between the test and induction lines because there are two luminance gradients associated with each of these visual contours. The luminance gradient bounding the inside of each line is slightly shorter than the luminance gradient bounding the outside. There are also two luminance gradients comprising each of the lines in figure B, but because of the greater line width here, the luminance gradient on the inside of each line is considerably shorter than the gradient on the outside, thus providing less contour information in this figure. In figure $\mathrm{C}$, the luminance gradient on the inside of each line is shorter yet, and provides substantially less contour information. Finally, in figure $D$, there is a single luminance gradient bounding the outside of the figure, the gradients inside the other figures having vanished altogether here. This progressive decrease in contour information should lead to greater confusion of the test and induction contours, and should produce increasing Bourdon illusions and unbending effects across the set of figures.

The feature model predicts the same order of these effects as does the cognitive model. Referring to Figure 2, consider the two lines in Panel B. If the widths of these lines were increased, keeping their outside boundaries at
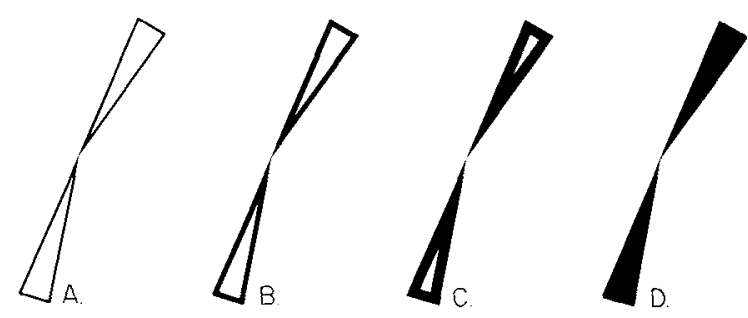

Figure 7. Real-contour illusions in Experiment 3. Line widths: (A) $2 \mathrm{~mm}$; (B) $4 \mathrm{~mm}$; (C) $8 \mathrm{~mm}$; (D) solid figure. 
a constant location, then their distributions of excitation and inhibition would approach - and eventually coincide with-the distributions shown for the edges in Panel D. Thus, to the extent that wider lines are more edge-like, the feature model predicts larger effects with increasing line width.

\section{Method}

Subjects. Seventeen introductory psychology students served as subjects: 9 men, 7 women, and 1 person whose gender was not recorded. Their mean age was 18.24 years, ranging from 18 to 24 .

Stimuli. The illusions were of the same size and orientation as in the previous experiments. The widths of the test and induction contours in the first three illusions were 2,4 , and $8 \mathrm{~mm}$; the fourth illusion was a solid figure.

Procedure. The procedures were the same as those used in the previous experiments

\section{Results and Discussion}

Figure 8 shows the results. The Bourdon illusion differed significantly from zero in all of the figures, and the unbending effect was significant in all but one, as the asterisks show.

A $2 \times 4$ analysis of variance was carried out. The factors were kinds of contours (test vs. induction) and line width $(2,4,8 \mathrm{~mm}$, and solid). There were repeated measures on both factors.

There was no significant main effect of contours $(F<1)$, and thus no difference overall between the Bourdon illusion and the unbending effect, consistent with the models. There was no main effect of line width $(F<1)$, contrary to predictions, but the contour $\times$ width interaction approached significance $[F(3,48)=2.39, p<.10]$. Thus, the simple effects of line width on the Bourdon illusion and the unbending effect are of interest. The simple effect of line width was significant for the Bourdon illusion $[F(3,93)=2.91, p<.05]$, and a trend analysis showed a significant linear component $[F(1,93)=8.71$, $p<.01]$. There was no significant simple effect of line width on the apparent unbending of the induction contours $(F<1)$.

The linear trend in the Bourdon illusion is consistent with both models, but the absence of a trend in the un-

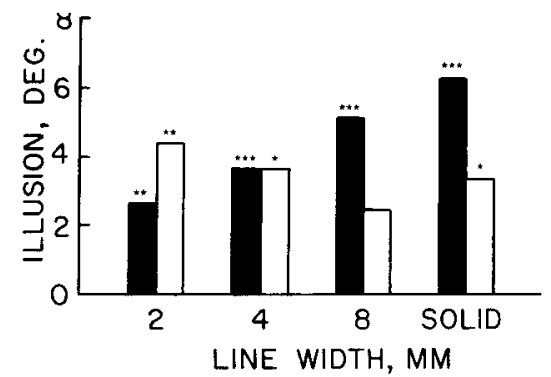

Figure 8. Results of Experiment 3. Bourdon illusion and unbending effect in real-contour figures. Significance levels: ${ }^{*} p<.05$, ${ }^{* * p}<.01,{ }^{* * *} p<.001$. bending effect is contrary to predictions. Thus, the present results provide limited support for our models.

The same illusion occurred in the comparison stimuli as in Experiments 1 and 2. The midpoints of the bent comparison lines appeared higher on the right side of the display to 14 of the 17 subjects $(Z=2.43, p<.02)$. Combining the results of the three experiments in the present study, 42 out of 50 subjects reported this illusion $(Z=4.67, p<.001)$.

\section{GENERAL DISCUSSION}

The results of Experiment 1 provide strong support for the cognitive model and the feature model as well, except in one respect. Neither model predicted the observed difference between the Bourdon illusion and the unbending effect. However, within each of these effects, both models predicted the observed order of the measurements across the four illusion figures, namely real lines $<$ real edges $<$ subjective lines $<$ subjective edges. The linear trends associated with this predicted order were highly significant.

In Experiment 2, the illusion figures were all defined by subjective lines and edges. The pattern of results was similar to the pattern observed in Experiment 1, although less orderly. The observed measurements of the Bourdon illusion were closer to the predicted order than were the measurements of the unbending effect. The linear trends associated with these orders were significant, but there were a few reversals in these trends. Contrary to prediction, the Bourdon illusion was greater than the unbending effect.

Experiment 3 measured the effects of different widths of real lines on the Bourdon illusion and the unbending effect. Consistent with the models, there was no overall difference between the Bourdon illusion and the unbending effect here. The Bourdon illusion measurements were in the predicted order across the set of figures, but the measurements of the unbending effect were not.

The results of all three experiments show that the mutual attraction between the test and induction contours is asymmetrical. Thus, it may be that different processes underlie the Bourdon illusion and the unbending effect. It is also possible that the different orientations of the test and induction contours are involved in this asymmetry.

Our test contours were oriented $22.5^{\circ}$ from the vertical. Rozvany and Day (1980) found the Bourdon illusion maximal at this orientation and much reduced when the test contours were vertical, or at an angle of $45^{\circ}$ from the vertical. Wenderoth and $O^{\prime}$ Connor (1987b) also found the greatest Bourdon illusion in a solid figure oriented $22.5^{\circ}$ from the vertical. Our upper and lower induction contours were oriented $35^{\circ}$ and $10^{\circ}$ from the vertical, respectively. It may be that any contour-attraction process-whether the Bourdon illusion or the unbending effect-would be reduced in a line or edge that had the orientation of one of our induction contours. Thus, the 
Bourdon illusion and the unbending effect might result from the same process, but differ in magnitude because of the different orientations of the collinear test contours and the bent induction contours.

In Experiments 1 and 3, the cognitive model and the feature model made identical predictions. In Experiment 2 , the cognitive model made unequivocal predictions, but the predictions of the feature model were not so clear. Overall, the predictions of the two models were essentially indistinguishable in the present study. Thus, none of the present results could have supported or disconfirmed either model independently of the other, although both could have been supported or both disconfirmed.

In other situations, the two models make differential predictions. The cognitive model predicts mutual attraction between the test and induction contours at all separations-at least within the range of angles usually employed in the Bourdon illusion. However, the feature model predicts repulsion between lines at some separations and attraction at smaller angles, and predicts only attraction between edges that are close enough to interact at all. Thus, under some conditions, there should be repulsion between lines at some particular separation where edges should attract each other. As we noted in the introduction, in some cases outline figures do, in fact, produce repulsion-that is, negative Bourdon illusions-at separations where attraction occurs in solid figures (Rozvany \& Day, 1980; Wenderoth \& O'Connor, 1987a, 1987b; Wenderoth et al., 1986a).

Based on their observations of repulsion in outline Bourdon illusions and single outline angles and attraction in solid figures, Wenderoth and O'Connor (1987b) argued that these effects had different determinants. They explained the repulsion in outlines in terms of lateral inhibition, and the attraction in solid figures as a failure to discriminate accurately between the judged edge, the other edge, and the bisector of the angle between these edges, invoking the oblique effect (Appelle, 1972; Rozvany \& Day, 1980) to explain the near absence of the Bourdon illusion in solid figures with horizontal or vertical test edges.

Wenderoth and his colleagues have consistently found repulsion in outline figures, whereas we have consistently found small attraction effects in figures with the same angular separation between test and induction contours. However, there are many differences between our experimental conditions and theirs, for example, in the size and luminance of the stimuli and, particularly, in the measurement methods. They used an adjustment procedure in which a comparison line was located $.3^{\circ}$ to $1.2^{\circ}$ to the upper left of the test contour. The comparison was adjusted in small increments of bending so as to appear bent in the same way as the test contour. The close proximity of their comparison line and test contours suggests the possibility of interactions between these stimuli, with unknown consequences.
Our measurement procedure used an array of fixed comparison stimuli centered $9.7^{\circ}$ below the center of each illusion. This greater separation may have decreased the likelihood of interactions between our comparison stimuli and the test or induction edges, but may also have increased the difficulty of the judgments. Nevertheless, the Bourdon illusions in our real outline and solid figures are closely comparable to the earlier results of Rozvany and Day (1980), who used an adjustment procedure where half of the figure was rotated to make the test lines or edges appear collinear.

Our cognitive model, based on the confusion of the test and induction contours, is very similar to Wenderoth and O'Connor's (1987b) explanation of attraction effects in solid figures resulting from failures of discrimination. Their explanation of repulsion in outlines, based on lateral inhibition, seems to leave little room for explaining the fact that outlines sometimes show attraction. Our feature model accounts for attraction and repulsion between lines, and for attraction between edges, in terms of a single fundamental process: interaction between feature detectors. It may be instructive to apply this model in other contexts involving interactions between lines and edges.

\section{REFERENCES}

APPELLE, S. (1972). Perception and discrimination as a function of stimulus orientation. Psychological Bulletin, 78, 266-278.

Bourdon, B. (1902). La perception visuelle de l'espace. Paris: Reinwald. Bradley, D. R., \& Mates, S. M. (1985). Perceptual organization and apparent brightness in subjective contour figures. Perception, 14, 645-653.

Bradley, D. R., \& Petry, H. M. (1977). Organizational determinants of subjective contour: The subjective Necker cube. American Journal of Psychology, 90, 253-262.

CoREN, S., \& GiRGus, J. S. (1978). Seeing is deceiving: The psychology of visual illusions. Hillsdale, NJ: Erlbaum.

DAY, R. H. (1962). Excitatory and inhibitory processses as the basis of contour shift and negative after-effect. Psychologia, 5, 185-193.

FARNE, M. (1968). Alcune osservazioni con linee virtuali e margini quasi percettivi. Bolletino della Societá Italiana di Biologia Sperimentale, 44, 1613-1616.

Frisby, J. P., Clatworthy, J. L. (1975). Illusory contours: Curious cases of simultaneous brightness contrast? Perception, 4, 349-357.

GANZ, L. (1966). Mechanism of the figural aftereffects. Psychological Review, 73, 128-150.

Gibson, J. J., \& RADNER, M. (1937). Adaptation, after-effect, and contrast in the perception of tilted lines: I. Quantitative studies. Journal of Experimental Psychology, 20, 453-467.

Goldstein, M. B., \& Weintraub, D. L. (1972). The parallel-less Poggendorff: Virtual contours put the illusion down but not out. Perception \& Psychophysics, 11, 353-355.

Gregory, R. L. (1972). Cognitive contours. Nature, 238, 51-52.

HALPERN, D., \& SALTZMAN, B. (1983). The multiple determination of illusory contours: 1. A review. Perception, 12, 281-291.

Halpern, D., Saltzman, B., Harrison, W., \& Widaman, K. (1983). The multiple determination of illusory contours: 2 . An empirical in vestigation. Perception, 12, 293-303.

Hubel, D. H., \& Wiesel, T. N. (1968). Receptive fields and functional architecture of monkey striate cortex. Journal of Physiology, 195, 215-243.

JASTROW, J. (1891). A study of Zöllner's figures and other related illusions. American Journal of Psychology, 4, 381-398. 
KANIZsA, G. (1955). Margini quasi-percettivi in campi con stimolazione omogenea. Rivista di Psicologia, 49, 7-30.

Kanizsa, G. (1979). Organization in vision. New York: Praeger.

LuCKIESH, M. (1965). Visual illusions. New York: Dover. (Original work published 1922)

Meyer, G. E., \& GARgES, C. (1979). Subjective contours and the Poggendorff illusion. Perception \& Psychophysics, 26, 302-304.

OSGOOD, C. E., \& HEYER, A. W. (1952). A new interpretation of figural aftereffects. Psychological Review, 59, 98-118.

PASTORE, N. (1971). Selective history of theories of visual perception. New York: Oxford University Press.

Pressey, A. W., \& DEN Heyer, K. (1968). Observations on Chiang's "new" theory of geometrical illusions. Perception \& Psychophysics, 4, 313-314.

RATLIFF, F. (1965). Mach bands: Quantitative studies on neural networks in the retina. New York: Holden-Day.

Rock, I., \& ANSON, R. (1979). Illusory contours as the solution to a problem. Perception, 8, 665-681.

Rozvany, G. I. N., \& DaY, R. H. (1980). Determinants of the Bourdon effect. Perception \& Psychophysics, 28, 39-44.

Smith, A., \& OVer, R. (1975). Tilt aftereffects with subjective contours. Nature, 257, 581-582.

VoGels, R., \& Orban, G. A. (1987). Illusory contour orientation determination. Vision Research, 27, 453-467.

von BÉKÉsy, G. (1967). Sensory inhibition. Princeton, NJ: Princeton University Press.
Walker, J. T., \& Shank, M. D. (1987). The Bourdon illusion in subjective contours. Perception \& Psychophysics, 42, 15-24.

WALKER, J. T., \& Shank, M. D. (in press). Interactions between real and subjective contours in the Bourdon illusion. Perception \& Psychophysics.

WARE, C. (1981). Subjective contours independent of subjective brightness. Perception \& Psychophysics, 29, 500-504.

WENDEROTH, P., \& O'CONNOR, T. (1987a). The effects of display and observer strategy variables on Bourdon assimilation illusions. Perception, 16, 79-88.

Wenderoth, P., \& O'ConNor, T. (1987b). Outline- and solid-angle orientation illusions have different determinants. Perception \& Psychophysics, 41, 45-52.

WENDEROTh, P., O'ConNor, T., \& JohnSON, M. (1986a). Expansion and contraction of outline and solid acute angles: Effects of angle magnitude, type of display, and the nature of the matching task. Perception \& Psychophysics, 39, 261-266.

WENDEROTH, P., O'CONNOR, T. O., JohnSON, M. (1986b). The tilt illusion as a function of the relative and absolute lengths of test and inducing lines. Perception \& Psychophysics, 39, 339-345.

WINER, B. J. (1971). Statistical principles in experimental design (2nd ed.). New York: McGraw-Hill.

(Manuscript received June 22, 1987;

revision accepted for publication November 19, 1987.) 